\title{
Clinical and Laboratory Features Associated with Acute Kidney Injury in Severe Malaria
}

\author{
Hiren Anghan, Prayas Sethi, Manish Soneja, Sandeep Mahajan', Naveet Wig \\ Departments of Medicine and 'Nephrology, All India Institute of Medical Sciences, New Delhi, India
}

\section{Abstract}

Introduction: Critically ill severe malaria constitutes one of the major hospital admissions in Indian setting. Clinical studies identifying the factors associated with acute kidney injury (AKI) in malaria are lacking. This study aimed to identify these factors. Methods: This prospective observational study was conducted in a tertiary care center of North India. All adult patients with severe malaria were studied during 2012-2014. Results: The study included 79 patients and AKI was observed in 36 patients. Of these 79 patients, 52.7\% were Plasmodium falciparum positive and $47.2 \%$ were Plasmodium vivax positive. In AKI patients, thrombocytopenia and jaundice were the most common other complications seen. Among P. vivax malarial patients, 17 (36\%) patients had AKI. Features associated with AKI among patients admitted with $P$. vivax malaria were as follows: tachycardia (adjusted relative risk [RR]: 3.9; 95\% confidence interval [CI]: 1.1-13.7), direct hyperbilirubinemia (adjusted RR: 4.7; 95\% CI: 1.4-15.2), anemia (adjusted RR: 6; 95\% CI: 1.7-22.4), and sepsis (adjusted RR: 3.3; 95\% CI: 1.1-13.7). The presence of tachycardia, acidosis, cerebral malaria, acute respiratory distress syndrome/acute lung injury, hypotensive shock, and poor Glasgow Coma Scale were associated with higher mortality in patients with AKI. Patients who required mechanical ventilation and/or vasopressor support had higher mortality. Conclusion: $P$. vivax is an important cause of severe malaria with AKI in our setting. Various other clinical features are associated with AKI and related mortality.

Keywords: Acute kidney injury, critically ill, severe malaria

\section{INTRODUCTION}

Tropical countries, including India, carry the major burden of malaria. Severe malaria is diagnosed by demonstrating asexual forms of the malaria parasites in the blood in a patient with a potentially fatal manifestation or complication of malaria in whom other diagnoses have been excluded. Our intensive care team deals routinely with malaria complications as they require critical care management. These complications, as defined by the WHO, include cerebral malaria, pulmonary edema, acute renal failure, acidosis, hypoglycemia, severe anemia, bleeding, jaundice, and shock. Recent evidence is that the overall burden, economic impact, and severity of Plasmodium vivax have been underestimated ${ }^{[1]}$

Malaria contributes $2 \%-39 \%$ to overall hospital admissions for acute kidney injury (AKI). In endemic areas, the incidence of AKI may be $>4 \%$ of malaria cases. Among those with severe parasitemia, as many as $60 \%$ develop AKI. Renal involvement has been reported in Plasmodium falciparum, Plasmodium

\begin{tabular}{|l|l|}
\hline \multicolumn{3}{|c|}{ Access this article online } \\
\hline Quick Response Code: & Website: \\
\hline & www.ijccm.org \\
\hline & \\
\hline
\end{tabular}

malariae, and recently in P. vivax infections. AKI is usually seen at the end of the $1^{\text {st }}$ week and is nonoliguric in $50 \%-75 \%{ }^{\left[{ }^{[2]}\right.}$ In view of the high mortality rates associated with AKI, it is critical to identify the clinical and laboratory parameters associated with AKI in a patient with malaria, to avoid progression to renal failure. Good hydration, monitoring of urine output, and renal function with careful use of nephrotoxic medications are the bedrock of supportive care. Timely transfer for dialysis is particularly important and is a challenging issue in resource-limited settings.

Our study was designed to estimate the incidence and analyze the parameters associated with AKI in severe malaria. Such data from Indian settings are scarce.

Address for correspondence: Prof. Naveet Wig, Room No 3097, Department of Medicine, All India Institute of Medical Sciences, New Delhi, India. E-mail: naveetwig@gmail.com

This is an open access journal, and articles are distributed under the terms of the Creative Commons Attribution-NonCommercial-ShareAlike 4.0 License, which allows others to remix, tweak, and build upon the work non-commercially, as long as appropriate credit is given and the new creations are licensed under the identical terms.

For reprints contact: reprints@medknow.com

How to cite this article: Anghan $\mathrm{H}$, Sethi $\mathrm{P}$, Soneja M, Mahajan S, Wig N. Clinical and laboratory features associated with acute kidney injury in severe malaria. Indian J Crit Care Med 2018;22:718-22. 


\section{Methods}

Ours was a prospective observational study conducted in a tertiary care center of North India from 2012 to 2014. All patients $>12$ years with confirmed severe malaria who were willing to participate were studied. A total of 64 severe malaria cases without AKI were needed to estimate the incidence with $95 \%$ confidence interval (CI) and $25 \%$ relative precision. Patients on nephrotoxic drugs, with other chronic diseases, or patients not giving consent were excluded from the study. Patients were followed up till a month after discharge or death. The diagnosis of malaria was made on all patients screened for acute febrile illness by a positive plasmodium lactate dehydrogenase antigen-based rapid diagnostic test and peripheral blood film confirmed on Giemsa stain.

Apart from the parasite density, percentage of parasitized red blood cells (parasitemia percentage) and the presence of one or more schizonts in the Giemsa-stained smear were also noted. Blood samples were taken on the day of admission for full blood count, renal function, liver function, glucose, and arterial blood gas analysis. A chest radiograph was obtained, and blood was collected for bacterial culture sensitivity, Widal test, HIV, hepatitis profile, NS1 antigen for dengue, Leptospira serology, and other specific tests (i.e., lumbar puncture) to exclude any other infection according to symptoms as clinically indicated.

AKI network (AKIN) criteria to define AKI in patients with severe malaria were used as these criteria are more sensitive and specific. It differs from RIFLE (Risk, injury, failure, loss and end stage kidney disease) classification, in that it reduces the need for baseline creatinine but requires two creatinine values within $48 \mathrm{~h} \cdot{ }^{[3]}$ Serum creatinine $1.5-2$ times from baseline or $25 \mu \mathrm{mol} / \mathrm{L}$ from baseline increase or urine output $<400 \mathrm{~mL} / 24 \mathrm{~h}$ or $<0.5 \mathrm{~mL} / \mathrm{kg} / \mathrm{h}$ were taken to be indicative of AKI. Renal replacement therapy was given in the form of hemodialysis as indicated.

Data were analyzed using STATA 12.1 software. Descriptive statistics for continuous variables were represented as mean \pm standard deviation as well as median and range (minimum to maximum). Categorical variables were reported in terms of the number and percentage of patients affected. The incidence of AKI was calculated with $95 \%$ CI in severe malaria. In case of continuous variable, $t$-test/Wilcoxon rank sum test was applied as appropriate to calculate the difference in average between the groups. The Chi-square test was used to calculate the association of categorical variables with outcome. Receiver operating characteristic curve analysis was done to find the cutoff of variables where not available. Univariate logistic regression and stepwise multiple logistic regression were applied to calculate the unadjusted and adjusted odds ratio (OR). This was consequently converted to relative risk (RR).

\section{RESULTS}

From the 100 patients screened, 79 with severe malaria were recruited. Among these, $47(59.5 \%)$ had $P$. vivax malaria and
$32(40.5 \%)$ had P. falciparum malaria. The most common associations were thrombocytopenia $(71,89.8 \%)$, jaundice (50, $63.2 \%$ ), and renal failure $(36,45.6 \%)$. Sepsis was present in $25(31.6 \%)$ patients. Cerebral malaria, severe anemia, and acute respiratory distress syndrome (ARDS) were present in $15(18.9 \%), 12(16 \%)$, and $10(12.6 \%)$, respectively.

Of the 36 patients with AKI, 19 (52.7\%) were P. falciparum positive compared to 17 (47.2\%) were P. vivax positive. None were positive for both P. falciparum and P. vivax. However, polymerase chain reaction-based tests were not performed in our study. Patients with AKI were classified into three stages on the basis of the AKIN classification. On the day of onset of AKI, $9(25 \%)$ were in Stage 1, 8 (22\%) in Stage 2, and $19(53 \%)$ in Stage 3. The final AKI stage recorded (i.e., the uppermost stage of AKI that developed before improvement began) were as follows: $4(13 \%)$ in Stage 1, $11(30 \%)$ in Stage 2, and 21 (57\%) in Stage 3.

The comparison between the clinical and laboratory parameters of AKI versus non-AKI group is shown in Table 1. On univariate analysis, vomiting was significantly higher in patients with AKI. Patients with AKI were more likely to have tachycardia ( $>96 / \mathrm{min})$, tachypnea ( $>20$ per $\mathrm{min})$, and pallor. Anemia (hemoglobin $[\mathrm{Hb}]<70 \mathrm{~g} / \mathrm{L}$ ), leukocytosis, levels of direct bilirubin $>1.5 \mathrm{mg} / \mathrm{dl}$, lactate $>5 \mathrm{mmol} / \mathrm{L}$, transaminase $>40 \mathrm{IU} / \mathrm{L}$, and infection with $P$. falciparum species were significantly associated with AKI. Sepsis was significantly more frequent in $18 / 36(50 \%)$ with AKI than in $7(16 \%)$ without.

On multivariate analysis, higher prevalence ratio (adjusted RR: 3.9; 95\% CI: 1.1-13.7), direct hyperbilirubinemia (adjusted RR: 4.7; 95\% CI: 1.4-15.2), lower $\mathrm{Hb}(<70 \mathrm{~g} / \mathrm{L})$ (adjusted RR: 6; 95\% CI: 1.7-21.4), and sepsis (adjusted RR: 3.3; 95\% CI: 1-10.5) were significantly associated with AKI.

Thrombocytopenia was seen in $33(91 \%)$ of those with and 38 (88\%) without AKI. Complications that were significantly higher in AKI included ARDS (OR: 5.85; 95\% CI: 1.85-29.66), sepsis (OR: 5.14; 95\% CI: 1.81-14.55), acidosis (OR: 4.9; 95\% CI: 1.66-14.5), and shock (OR: 2.69; 95\% CI: 1-7.25). Comparing the two species, 14 (78\%) P. falciparum patients with AKI required dialysis as compared to $6(35 \%)$ with $P$. vivax; this difference was statistically significant $(P=0.02)$.

A total of 11/79 severe malaria patients died. Features on admission associated with a fatal outcome are shown in Table 2.

The association of different complications of malaria in AKI patients with mortality is shown in Table 3 .

Acidosis $(P=0.001)$, sepsis $(P=0.016)$, and renal failure $(P=0.042)$ were more common in $P$. falciparum than $P$. vivax malaria. Several patients with $P$. vivax malaria also had these complications. No patient had a mixed infection with both $P$. vivax and $P$. falciparum. The frequency of various complications in the two types of malaria is shown in Table 4. 


\begin{tabular}{|c|c|c|c|c|}
\hline Variable & With AKI, frequency (\%) $(n=36)$ & Without AKI, frequency (\%) $(n=43)$ & $P$ & Univariate RR (95\% CI) \\
\hline \multicolumn{5}{|l|}{ Pulse rate } \\
\hline$>96$ & $25(59.5)$ & $17(39.5)$ & 0.008 & $3.5(1.4-8.9)$ \\
\hline \multicolumn{5}{|l|}{ Respiratory rate } \\
\hline$>20$ & $30(83.4)$ & $19(44.2)$ & 0.001 & $6.3(2.2-18.2)$ \\
\hline \multicolumn{5}{|l|}{ Nausea and vomiting } \\
\hline Yes & $32(88.9)$ & $30(69.8)$ & 0.039 & $3.5(1.01-11.8)$ \\
\hline \multicolumn{5}{|l|}{ Pallor } \\
\hline Yes & $27(75)$ & $22(51.5)$ & 0.030 & $2.9(1.1-7.5)$ \\
\hline \multicolumn{5}{|l|}{ Hemoglobin (g/l) } \\
\hline$>70$ & $19(52.7)$ & $34(79.1)$ & 0.012 & $3.38(1.2-9)$ \\
\hline \multicolumn{5}{|l|}{ Conjugated bilirubin } \\
\hline$>26.6$ & $24(66.7)$ & $15(34.8)$ & 0.007 & $3.7(1.5-9.5)$ \\
\hline \multicolumn{5}{|l|}{ SGOT } \\
\hline$>40$ & $23(64)$ & $12(28)$ & 0.002 & $4.6(1.7-11.8)$ \\
\hline \multicolumn{5}{|l|}{ Lactate } \\
\hline$>5$ & $14(39)$ & $7(16.8)$ & 0.023 & $3.3(1.1-9.3)$ \\
\hline \multicolumn{5}{|l|}{ Species } \\
\hline Plasmodium falciparum & $19(47.2)$ & $13(30)$ & 0.042 & $2.6(1.02-6.5)$ \\
\hline \multicolumn{5}{|l|}{ Sepsis } \\
\hline Yes & $18(50)$ & $7(16)$ & 0.001 & $5.1(1.8-14.5)$ \\
\hline
\end{tabular}

Univariate analysis (values expressed in terms of RR with $95 \%$ of $\mathrm{CI}$ on univariate analysis). AKI: Acute kidney injury; CI: Confidence interval; RR: Relative risk; SGOT: Serum glutamic-oxaloacetic transaminase

Table 2: Statistically significant "clinical features in acute kidney injury fatalities"

\begin{tabular}{lccc}
\hline Variables & AKI died $(\boldsymbol{n = 9 )}$ & AKI survived, $(\boldsymbol{n = 2 7 )}$ & $\boldsymbol{P}$ \\
\hline PR (per min), mean \pm SD & $112.4 \pm 4.8$ & $101.9 \pm 11.1$ & 0.01 \\
SBP (mm Hg), mean \pm SD & $82.4 \pm 6.2$ & $99 \pm 14$ & 0.009 \\
TLC (per cu mm) & $13,000(6100-25,000)$ & $9000(1500-23,000)$ & 0.03 \\
GCS, mean \pm SD & $7.1 \pm 3.8$ & $14.2 \pm 1.5$ & 0.001 \\
APACHE II score & $26.2 \pm 7$ & $11.9 \pm 5.8$ & 0.002 \\
Mechanical ventilation (\%) & $7(78)$ & $4(14)$ & 0.001 \\
Vasopressor support (\%) & $7(78)$ & $4(14)$ & 0.001 \\
\hline AKI Acu
\end{tabular}

AKI: Acute kidney injury; SD: Standard deviation; PR: Prevalence ratio; SBP: Systolic blood pressure; GCS: Glasgow Coma Scale; APACHE: Acute Physiology and Chronic Health Evaluation; TLC: Total leucocyte count

\section{Discussion}

$P$. falciparum has been a major cause of complicated malaria, but over the years, there have been numerous cases of $P$. vivax malaria- causing severe complications. The disturbing fact is that the most common and widespread species of malaria is $P$. vivax. The exact pathogenesis, parasite-host interactions, and reasons for multi-organ dysfunction due to $P$. vivax are unclear. ${ }^{[4]}$

In our study, 47 (59.49\%) patients had $P$. vivax malaria who satisfied the WHO criteria for severe malaria indicating the underestimated severe presentation of $P$. vivax malaria. The presence of $P$. falciparum malaria has not shown statistically significant difference in risk of increased mortality as compared to $P$. vivax, and even though the falciparum has shown statistically significant increased chances of complications associated with malaria, the absolute number of patients with these complications is high. This is consistent with the globally increased complicated $P$. vivax malaria. In a systematic review and meta-analysis by Rahimi et al., on severe $P$. vivax malaria, there has been an increasing trend in the prevalence of complications in $P$. vivax malaria. ${ }^{[5]}$ Among the 77 studies reporting severe $P$. vivax malaria, severe thrombocytopenia $\left(<50,000 / \mathrm{mm}^{3}\right)$ was the most common "severe" manifestation $(888 / 45,775$ with pooled prevalence of $8.6 \%)$. The case fatality was $0.3 \%(353 / 46,411)$. Likewise, there have been various isolated case reports and series on fatalities seen in $P$. vivax malaria.

Numerous criteria for diagnosing AKI are available such as the WHO, RIFLE, and AKIN. AKIN criteria for AKI was used keeping in view that small change in serum creatinine is associated with adverse outcomes, and consequent to its increased sensitivity, it estimated the AKI prevalence of $45 \%$ which is higher than the studies done earlier. Different studies have shown variable prevalence of AKI in malaria ranging from $6 \%$ to $40 \%$. Ratanarat et al. found that AKIN criteria improved sensitivity for detection of AKI, and prediction of inhospital mortality was better than that of RIFLE criteria in critically ill patients with multi-organ dysfunction syndrome. ${ }^{[6]}$ 


\begin{tabular}{|c|c|c|c|c|}
\hline Complications & Died $(n=9)$ & Survived $(n=27)$ & $P$ & OR (95\% Cl) \\
\hline \multicolumn{5}{|l|}{ Cerebral malaria } \\
\hline Yes & 6 & 4 & 0.012 & $11.5(2.007-65.9)$ \\
\hline \multicolumn{5}{|l|}{ ARDS/ALI } \\
\hline Yes & 7 & 2 & 0.002 & $43.7(5.91-368.7)$ \\
\hline \multicolumn{5}{|l|}{ Acidosis } \\
\hline Yes & 7 & 9 & 0.049 & $7(1.2-40.82)$ \\
\hline \multicolumn{5}{|l|}{ Sepsis } \\
\hline Yes & 8 & 10 & 0.018 & $13.6(1.47-143.5)$ \\
\hline \multicolumn{5}{|c|}{ Hypotensive shock } \\
\hline Yes & 2 & 7 & 0.004 & $28(2.89-270.5)$ \\
\hline \multicolumn{5}{|l|}{ Poor GCS } \\
\hline Yes & 6 & 1 & $<0.001$ & $52(4.57-591)$ \\
\hline
\end{tabular}

\begin{tabular}{|c|c|c|c|c|}
\hline Complications & $\begin{array}{l}\text { Plasmodium falciparum } \\
\text { frequency (percentage of } n=32 \text { ) }\end{array}$ & $\begin{array}{l}\text { Plasmodium vivax frequency } \\
\text { (percentage of } n=47 \text { ) }\end{array}$ & $P$ & OR (95\% CI) \\
\hline \multicolumn{5}{|l|}{ Hyperpyrexia } \\
\hline Yes & $13(40.7)$ & $19(40.4)$ & 0.98 & $1(0.4-2.25)$ \\
\hline \multicolumn{5}{|l|}{ Cerebral malaria } \\
\hline Yes & $9(28.1)$ & $6(12.8)$ & 0.08 & $3.12(0.62-15.69)$ \\
\hline \multicolumn{5}{|l|}{ ARDS/ALI } \\
\hline Yes & $5(15.6)$ & $6(12.8)$ & 0.71 & $0.41(0.07-2.39)$ \\
\hline \multicolumn{5}{|l|}{ Jaundice } \\
\hline Yes & $21(65.3)$ & $31(66)$ & 0.97 & $0.9(0.3-2.66)$ \\
\hline \multicolumn{5}{|l|}{ Renal failure } \\
\hline Yes & $19(59.3)$ & $17(36.1)$ & 0.042 & $2.57(1.02-6.49)$ \\
\hline \multicolumn{5}{|l|}{ Severe anemia } \\
\hline Yes & $7(21.9)$ & $5(10.6)$ & 0.17 & $2.32(0.57-9.40)$ \\
\hline \multicolumn{5}{|l|}{ Bleeding/DIC } \\
\hline Yes & $12(37.5)$ & $15(31.9)$ & 0.60 & $0.88(0.28-2.77)$ \\
\hline \multicolumn{5}{|l|}{ Rash } \\
\hline Yes & $3(9.3)$ & $3(6.3)$ & 0.62 & $1.44(0.23-8.90)$ \\
\hline \multicolumn{5}{|c|}{ Thrombocytopenia } \\
\hline Yes & $27(84.38)$ & $44(93.6)$ & 0.18 & $0.32(0.06-1.62)$ \\
\hline \multicolumn{5}{|l|}{ Acidosis } \\
\hline Yes & $16(50)$ & $6(12.8)$ & 0.001 & $7.06(2.22-22.50)$ \\
\hline \multicolumn{5}{|c|}{ Hypotensive shock } \\
\hline Yes & $11(34.4)$ & $9(19.1)$ & 0.12 & $2.25(0.49-10.25)$ \\
\hline \multicolumn{5}{|l|}{ Sepsis } \\
\hline Yes & $15(46.9)$ & $10(21.2)$ & 0.016 & $3.26(1.21-8.74)$ \\
\hline \multicolumn{5}{|l|}{ Seizure } \\
\hline Yes & $3(9.3)$ & $2(4.2)$ & 0.39 & $2.5(0.39-15.92)$ \\
\hline \multicolumn{5}{|l|}{$>5 \%$ parasitemia } \\
\hline Yes & 0 & 0 & - & - \\
\hline \multicolumn{5}{|l|}{ Poor GCS } \\
\hline Yes & $5(15.6)$ & $5(10.6)$ & 0.52 & $0.41(0.05-3.42)$ \\
\hline
\end{tabular}

A systematic review and meta-analysis by Xiong et al. found that the AKIN criteria can identify more patients in classifying AKI in intensive care unit (ICU) patients compared to RIFLE criteria; however, the AKIN criteria did not show a better ability in predicting hospital mortality in both ICU and cardiac surgery patients compared to RIFLE criteria. ${ }^{[7]}$

Tachycardia, direct hyperbilirubinemia, and anemia $(<7 \mathrm{~g} / \mathrm{dl})$ were associated with AKI. The etiology of AKI in the presence 
of these factors can be attributed to hemolysis, parasite load, dehydration and fever per se, sepsis and Disseminated intravascular coagulation (DIC), etc. Cytokine response to severe infection could also be contributory. Hence, an anemic and icteric acute febrile patient in endemic area who tests positive for malaria merits serial monitoring of kidney functions. Severe anemia with $\mathrm{Hb}<5 \mathrm{~g} / \mathrm{dL}$ and/or hematocrit $<15 \%$ is one of the well-known complications and poor predictor of severe malaria. Apart from the WHO criteria for severe malaria, this predictive value of $\mathrm{Hb}$ and hematocrit level was also reported in studies done by Mishra et al. and von Seidlein et al. ${ }^{[8,9]}$ The present study has also shown the statistically significant value of low $\mathrm{Hb}$ level and hematocrit level in patients of AKI in severe malaria. In a study done by Saravu et al. for risk factors and outcome stratified by severity of AKI showed, increasing age, higher heart rate, lower diastolic blood pressure, icterus, and hepatomegaly were identified risk factors of AKI. ${ }^{[10]}$

The overall mortality rate among those with kidney injury ranges from $15 \%$ to $50 \%$ in different series. ${ }^{[11]} \mathrm{A}$ total of $11 / 79$ severe malaria patients died in our study which is almost in accord with the worldwide mortality rate.

The presence of tachycardia, acidosis, cerebral malaria, ARDS/acute lung injury, hypotensive shock, and poor Glasgow Coma Scale (GCS) was associated with higher mortality in patients with AKI. Patients who required mechanical ventilation and/or vasopressor support had higher mortality. It is needless to say that these conditions are associated with poor outcomes in all critically ill patients, and the presence of AKI can worsen these conditions. AKI can contribute to encephalopathy due to uremia, metabolic acidosis can be worsened, and superadded infections can worsen sepsis and may lead to ARDS. The presence of cerebral malaria is the main cause behind the low GCS score or impaired sensorium. Other factors that can cause similar poor sensorium are presence of hypoglycemia, electrolyte imbalance, and severe shock. The significant predictive value of low GCS score in severe malaria was also shown in a study done by Hanson et al.$^{[12]}$ They also developed a scoring system Coma, Acidosis, Malaria (CAM score) using this parameter as one of the components. The low GCS score was also one of the significant predictors of mortality in a study done by Dondorp et al.$^{[13]}$ Studies by Tangpukdee et al. in Thailand on adult malaria patients showed tachycardia as a predictor of mortality. ${ }^{[14]}$ Frequent presence of severe anemia and shock in patients who died may have contributed to the significant difference of pulse rate in the two study groups in our study.

\section{Strength and limitations of study}

This is probably the only study done using AKIN criteria to define AKI in malaria. However, the sample size was small with a referral bias.

Only admitted patients with suspected severe malaria were studied and that the size of denominator populations of people with $P$. falciparum and $P$. vivax infections was unknown (those not reaching a health facility; those managed in outpatients clinic; and those admitted but not severe) nor the proportion of them that reached hospital and were admitted.

\section{Conclusion}

The prevalence of AKI among patients admitted to hospital with severe malaria was $45.6 \%$ in our study. Direct hyperbilirubinemia, anemia, tachycardia, and sepsis were associated risk factors in these patients. This information can be applied in any resource-limited health-care setting at primary health-care level to identify patients who have a high likelihood of AKI in the presence of severe malaria and can be timely referred.

Future studies should investigate if even earlier detection with novel biomarkers, before rise in serum creatinine, would alter the epidemiology of this preventable complication. The present study shows that there is rising trend of complicated $P$. vivax malaria.

Financial support and sponsorship

Nil.

\section{Conflicts of interest}

There are no conflicts of interest.

\section{RefEREnCES}

1. Lacerda MV, Mourão MP, Alexandre MA, Siqueira AM, Magalhães BM, Martinez-Espinosa FE, et al. Understanding the clinical spectrum of complicated Plasmodium vivax malaria: A systematic review on the contributions of the Brazilian literature. Malar J 2012;11:12.

2. Sitprija V. Nephropathy in falciparum malaria. Kidney Int 1988;34:867-77.

3. Mehta RL, Kellum JA, Shah SV, Molitoris BA, Ronco C, Warnock DG, et al. Acute kidney injury network: Report of an initiative to improve outcomes in acute kidney injury. Crit Care 2007;11:R31.

4. Mehndiratta S, Rajeshwari K, Dubey AP. Multiple-organ dysfunction in a case of Plasmodium vivax malaria. J Vector Borne Dis 2013;50:71-3.

5. Rahimi BA, Thakkinstian A, White NJ, Sirivichayakul C, Dondorp AM, Chokejindachai W. Severe vivax malaria: A systematic review and meta-analysis of clinical studies since 1900. Malar J 2014;13:481.

6. Ratanarat R, Skulratanasak P, Tangkawattanakul N, Hantaweepant C. Clinical accuracy of RIFLE and acute Kidney Injury Network (AKIN) criteria for predicting hospital mortality in critically ill patients with multi-organ dysfunction syndrome. J Med Assoc Thai 2013;96 Suppl 2:S224-31.

7. Xiong J, Tang X, Hu Z, Nie L, Wang Y, Zhao J, et al. The RIFLE versus AKIN classification for incidence and mortality of acute kidney injury in critical ill patients: A meta-analysis. Sci Rep 2015;5:17917.

8. Mishra SK, Panigrahi P, Mishra R, Mohanty S. Prediction of outcome in adults with severe falciparum malaria: A new scoring system. Malar J 2007;6:24.

9. von Seidlein L, Olaosebikan R, Hendriksen IC, Lee SJ, Adedoyin OT, Agbenyega T, et al. Predicting the clinical outcome of severe falciparum malaria in African children: Findings from a large randomized trial. Clin Infect Dis 2012;54:1080-90.

10. Saravu K, Rishikesh K, Parikh CR. Risk factors and outcomes stratified by severity of acute kidney injury in malaria. PLoS One 2014;9:e90419.

11. Kute VB, Trivedi HL, Vanikar AV, Shah PR, Gumber MR, Patel HV, et al. Plasmodium vivax malaria-associated acute kidney injury, India, 2010-2011. Emerg Infect Dis 2012;18:842-5.

12. Hanson J, Lee SJ, Mohanty S, Faiz MA, Anstey NM, Charunwatthana P, et al. A simple score to predict the outcome of severe malaria in adults. Clin Infect Dis 2010;50:679-85.

13. Dondorp AM, Lee SJ, Faiz MA, Mishra S, Price R, Tjitra E, et al. The relationship between age and the manifestations of and mortality associated with severe malaria. Clin Infect Dis 2008;47:151-7.

14. Tangpukdee N, Duangdee C, Wilairatana P, Krudsood S. Malaria diagnosis: A brief review. Korean J Parasitol 2009;47:93-102. 